Henryk KoŁakowski and JarosŁaw Łazuka (Warszawa)

\title{
THE CAUCHY PROBLEM FOR THE SYSTEM OF PARTIAL DIFFERENTIAL EQUATIONS DESCRIBING NONSIMPLE THERMOELASTICITY
}

Abstract. The aim of this paper is to derive a formula for the solution to the Cauchy problem for the linear system of partial differential equations describing nonsimple thermoelasticity. Some properties of the solution are also presented. It is a first step to study the nonlinear case.

The theory of nonsimple elastic and thermoelastic materials was studied in various papers. R. A. Toupin [4] derived for the first time the equations of motion, constitutive equations and boundary conditions of the straingradient theory in general nonlinear form. On the basis of the conservation principle, R. D. Mindlin and N. N. Eshel [2] obtained the linear theory of elasticity in which the potential energy density depends not only on the strain but also on the gradient of the strain. G. Ahmadi and K. Firoozbakhsh [1] derived the strain-gradient theory of thermoelasticity based on the ClausiusDuhem inequality.

In this paper we consider the following initial value problem:

$$
u_{t t}+c_{2}^{2} l_{2}^{2} \Delta^{2} u+\left(c_{1}^{2} l_{1}^{2}-c_{2}^{2} l_{2}^{2}\right) \nabla \operatorname{div} \Delta u
$$

$$
\theta_{t}-\frac{k}{c} \Delta \theta+\frac{T_{0} m}{c} \nabla \cdot u_{t}=g \text { for } t>0,
$$

$$
+\frac{m}{\varrho} \nabla \theta-c_{2}^{2} \Delta u-\left(c_{1}^{2}-c_{2}^{2}\right) \nabla \operatorname{div} u=f,
$$

$$
u(0, x)=u^{0}(x), \quad u_{t}(0, x)=u^{1}(x), \quad \theta(0, x)=\theta_{0}(x),
$$

where $x \in \mathbb{R}^{3}, u: \overline{\mathbb{R}}_{+} \times \mathbb{R}^{3} \rightarrow \mathbb{R}^{3}, \theta: \overline{\mathbb{R}}_{+} \times \mathbb{R}^{3} \rightarrow \mathbb{R}, u$ denotes the displacement, $\theta$ the temperature disturbance, $f$ is a given vector-valued function on $[0, \infty) \times \mathbb{R}^{n}$ and $g$ is a given function also on $[0, \infty) \times \mathbb{R}^{n}$; $c_{1}, c_{2}, l_{1}, l_{2}, m, k, c, \varrho, T_{0}$ are some constant physical parameters.

2000 Mathematics Subject Classification: Primary 35Q72.

Key words and phrases: Cauchy problem, thermoelasticity, Sobolev spaces. 
The main results of this paper are the exact formula for the solution $(u, \theta)$ of the Cauchy problem (1)-(2) and the following theorem:

Theorem 1. Let $u^{0}, u^{1}, \theta_{0}$ be in the Sobolev space $H^{s+1}\left(\mathbb{R}^{3}\right)$ with norm $\|\cdot\|_{s+1}$ and $f, g \in C\left([0, t], H^{s+1}\left(\mathbb{R}^{3}\right)\right)$. Then the problem $(1)-(2)$ has a unique solution $u \in C^{2}\left([0, t], H^{s}\left(\mathbb{R}^{3}\right)\right), \theta \in C^{1}\left([0, t], H^{s}\left(\mathbb{R}^{3}\right)\right)$. This solution satisfies, for some constant $C_{1}$, the estimates

$$
\begin{aligned}
\|u\|_{s}^{2} \leq & C_{1}\left(1+t^{4}\right)\left(\left\|u^{0}\right\|_{s}^{2}+\left\|u^{1}\right\|_{s}^{2}+\left\|\theta_{0}\right\|_{s+1}^{2}\right. \\
& \left.+\sup _{\tau \in[0, t]}\|f(\tau)\|_{s}^{2}+\sup _{\tau \in[0, t]}\|g(\tau)\|_{s+1}^{2}\right), \\
\|\theta\|_{s}^{2} \leq & C_{1}\left(1+t^{4}\right)\left(\left\|u^{0}\right\|_{s+1}^{2}+\left\|u^{1}\right\|_{s+1}^{2}+\left\|\theta_{0}\right\|_{s}^{2}\right. \\
& \left.+\sup _{\tau \in[0, t]}\|f(\tau)\|_{s+1}^{2}+\sup _{\tau \in[0, t]}\|g(\tau)\|_{s+1}^{2}\right) .
\end{aligned}
$$

Now we derive the formulae for the Fourier transform of the solution to the Cauchy problem (1)-(2). Let $u_{t}=v$. Then the system (1) can be rewritten in the form

$$
\begin{aligned}
u_{t}= & v, \\
v_{t}= & -c_{2}^{2} l_{2}^{2} \Delta^{2} u-\left(c_{1}^{2} l_{1}^{2}-c_{2}^{2} l_{2}^{2}\right) \nabla \operatorname{div} \Delta u-\frac{m}{\varrho} \nabla \theta+c_{2}^{2} \Delta u \\
& +\left(c_{1}^{2}-c_{2}^{2}\right) \nabla \operatorname{div} u+f, \\
\theta_{t}= & \frac{k}{c} \Delta \theta-\frac{T_{0} m}{c} \nabla \cdot v+g,
\end{aligned}
$$

or briefly

$$
V_{t}=P\left(D_{x}\right) V+F
$$

where

$$
D_{x_{j}}=\frac{1}{i} \frac{\partial}{\partial_{x_{j}}}, \quad V=[u, v, \theta]^{T}, \quad F=[0, f, g]^{T} .
$$

We denote by $P\left(D_{x}\right)$ the differential operator with symbol $P(\xi)$. It can be shown that

$$
\lambda I-P(\xi)=\left[\begin{array}{ccccccc}
\lambda & 0 & 0 & -1 & 0 & 0 & 0 \\
0 & \lambda & 0 & 0 & -1 & 0 & 0 \\
0 & 0 & \lambda & 0 & 0 & -1 & 0 \\
a_{11} & a_{12} & a_{13} & \lambda & 0 & 0 & \beta \xi_{1} \\
a_{21} & a_{22} & a_{23} & 0 & \lambda & 0 & \beta \xi_{2} \\
a_{31} & a_{32} & a_{33} & 0 & 0 & \lambda & \beta \xi_{3} \\
0 & 0 & 0 & \alpha \xi_{1} & \alpha \xi_{2} & \alpha \xi_{3} & \lambda+D
\end{array}\right],
$$

where

$$
\left\{a_{i j}\right\}=a_{2} I+b \xi \otimes \xi \quad\left(\xi \otimes \xi=\left\{\xi_{i} \xi_{j}\right\}\right),
$$




$$
\begin{gathered}
a_{k}=c_{k}^{2} l_{k}^{2}|\xi|^{4}+c_{k}^{2}|\xi|^{2}, \quad b=\left(c_{1}^{2} l_{1}^{2}-c_{2}^{2} l_{2}^{2}\right)|\xi|^{2}+\left(c_{1}^{2}-c_{2}^{2}\right), \\
\beta=i \frac{m}{\varrho}, \quad \alpha=i \frac{T_{0} m}{c}, \quad D=\frac{k}{c}|\xi|^{2} .
\end{gathered}
$$

The characteristic polynomial of the matrix $P(\xi)$ has the form

$$
\operatorname{det}[\lambda I-P(\xi)]=\left[\lambda^{3}+D \lambda^{2}+\left(a_{1}+C\right) \lambda+D a_{1}\right]\left(\lambda^{2}+a_{2}\right)^{2},
$$

where $C=\frac{m^{2} T_{0}}{\varrho c}|\xi|^{2}$. It will be shown that the polynomial

$$
\kappa(\lambda)=\lambda^{3}+D \lambda^{2}+\left(a_{1}+C\right) \lambda+D a_{1}
$$

does not have multiple roots for all $\xi$ except possibly at most twelve values of $|\xi|>0$, and the roots have negative real parts (for $\xi \neq 0$ ). Let $w=\operatorname{rot} u$. Applying the rotation operator to the first equation of the system (1) we get the equation

$$
w_{t t}+c_{2}^{2} l_{2}^{2} \Delta^{2} w-c_{2}^{2} \Delta w=\operatorname{rot} f
$$

with initial data

$$
w(0, x)=\operatorname{rot} u^{0}(x), \quad w_{t}(0, x)=\operatorname{rot} u^{1}(x) .
$$

Thus for the Fourier transform of $w$ with respect to the spatial variables we obtain the Cauchy problem

$$
\begin{aligned}
& \widehat{w}_{t t}+a_{2} \widehat{w}=\widehat{\operatorname{rot} f} \quad \text { for } t>0, \\
& \widehat{w}=\widehat{\operatorname{rot} u^{0}}, \quad \widehat{w}_{t}=\widehat{\operatorname{rot} u^{1}} \quad \text { for } t=0 .
\end{aligned}
$$

The initial-value problem (6) has, for appropriate functions $f, u^{0}, u^{1}$, the unique solution

$$
\widehat{w}=\widehat{\operatorname{rot} u^{0}} \cos \sqrt{a_{2}} t+\widehat{\frac{\operatorname{rot} u^{1}}{\sqrt{a_{2}}}} \sin \sqrt{a_{2}} t+\int_{0}^{t} \frac{\sin \left[\sqrt{a_{2}}(t-s)\right] \widehat{\operatorname{rot} f(s)}}{\sqrt{a_{2}}} d s .
$$

Now, when applying the divergence operator to the first equation of the system (1) and using the notation $e=\operatorname{div} u$, we obtain the system

$$
\begin{gathered}
e_{t t}+c_{2}^{2} l_{2}^{2} \Delta^{2} e+\left(c_{1}^{2} l_{1}^{2}-c_{2}^{2} l_{2}^{2}\right) \Delta^{2} e+\frac{m}{\varrho} \Delta \theta-c_{2}^{2} \Delta e-\left(c_{1}^{2}-c_{2}^{2}\right) \Delta e=\operatorname{div} f \\
\theta_{t}-\frac{k}{c} \Delta \theta+\frac{T_{0} m}{c} e_{t}=g \quad(e=\operatorname{div} u) .
\end{gathered}
$$

Hence

$$
\begin{gathered}
e_{t t}+c_{1}^{2} l_{1}^{2} \Delta^{2} e-c_{1}^{2} \Delta e+\frac{m}{\varrho} \Delta \theta=\operatorname{div} f \\
\theta_{t}-\frac{k}{c} \Delta \theta+\frac{T_{0} m}{c} e_{t}=g .
\end{gathered}
$$


Applying the Fourier transform with respect to the spatial variables we get

$$
\begin{gathered}
\widehat{e}_{t t}+c_{1}^{2} l_{1}^{2}|\xi|^{4} \widehat{e}-\frac{m}{\varrho}|\xi|^{2} \widehat{\theta}+c_{1}^{2}|\xi|^{2} \widehat{e}=\widehat{\operatorname{div} f}, \\
\widehat{\theta}_{t}+\frac{k}{c}|\xi|^{2} \widehat{\theta}+\frac{T_{0} m}{c} \widehat{e}_{t}=\widehat{g} .
\end{gathered}
$$

Let $\widehat{e}_{t}=\widehat{v}$. Then

$$
\begin{aligned}
& \widehat{e}_{t}=\widehat{v}, \\
& \widehat{v}_{t}=-a_{1} \widehat{e}+\frac{m}{\varrho}|\xi|^{2} \widehat{\theta}+\widehat{\operatorname{div} f}, \\
& \widehat{\theta}_{t}=-\frac{k}{c}|\xi|^{2} \widehat{\theta}-\frac{T_{0} m}{c} \widehat{v}+\widehat{g} .
\end{aligned}
$$

This system can be expressed in the simple form

$$
Y_{t}=A Y+G,
$$

where $Y=[\widehat{e}, \widehat{v}, \widehat{\theta}]^{T}, G=[0, \widehat{\operatorname{div} f}, \widehat{g}]^{T}$, and $A$ is the matrix of coefficients. It can be easily seen that

$$
\operatorname{det}[\lambda I-A]=\kappa(\lambda)=\lambda^{3}+D \lambda^{2}+\left(a_{1}+C\right) \lambda+D a_{1} .
$$

The polynomial $\kappa(\lambda)$ is stable, i.e. all its roots have negative real parts (for $\xi \neq 0)$. This follows from [3, Theorem 6]. According to that theorem, if all principal minors of the matrix

$$
\left[\begin{array}{ccc}
d_{1} & d_{3} & 0 \\
d_{0} & d_{2} & 0 \\
0 & d_{1} & d_{3}
\end{array}\right]
$$

are positive, then the polynomial $d_{0} \lambda^{3}+d_{1} \lambda^{2}+d_{2} \lambda+d_{3}$ with $d_{0}>0$ is stable. Clearly, for the polynomial $\kappa(\lambda)$ this condition is fulfilled. The polynomial $\kappa(\lambda)$ has three distinct roots (almost everywhere with respect to $\xi \in \mathbb{R}^{3}$ ). Indeed, put $\lambda=z-\frac{1}{3} D$ into $\kappa(\lambda)$. We obtain

$$
z^{3}+p z+q
$$

where

$$
p=a_{1}+C-\frac{1}{3} D^{2}, \quad q=D-\frac{1}{3} D\left(a_{1}+C\right)+\frac{2}{27} D^{3} .
$$

Recall that the polynomial $z^{3}+p z+q$ and also $\kappa(\lambda)$ has multiple roots iff

$$
4 p^{3}+27 q^{2}=0 .
$$

The last equation as an equation of order 12 (with respect to $|\xi| \neq 0$ ) has at most 12 positive roots. Putting $G=0$ in the system (8) we obtain the following initial-value problem:

$$
Y_{t}=A Y, \quad Y(0)=\left[\widehat{e}_{0}, \widehat{v}_{0}, \widehat{\theta}_{0}\right]^{T} .
$$


The solution of this problem is

$$
Y=c_{1} Y_{1}+c_{2} Y_{2}+c_{3} Y_{3},
$$

where

$$
\begin{gathered}
Y_{k}=\left[\frac{\lambda_{k}\left(D+\lambda_{k}\right)+\tau \mu}{\mu a_{1}},-\frac{\left(D+\lambda_{k}\right)}{\mu}, 1\right]^{T} e^{\lambda_{k} t}, \\
\tau=\frac{m}{\varrho}|\xi|^{2}, \quad \mu=\frac{T_{0} m}{c} .
\end{gathered}
$$

The coefficients in (9) can be calculated by using Viète's formulas:

$$
\begin{aligned}
& c_{1}=\frac{\mu}{\left(\lambda_{2}-\lambda_{1}\right)\left(\lambda_{3}-\lambda_{1}\right)}\left[a_{1} \widehat{e}_{0}-\lambda_{1} \widehat{v}_{0}+\frac{\lambda_{1}^{2}+a_{1}}{\mu} \widehat{\theta}_{0}\right], \\
& c_{2}=\frac{-\mu}{\left(\lambda_{2}-\lambda_{1}\right)\left(\lambda_{3}-\lambda_{2}\right)}\left[a_{1} \widehat{e}_{0}-\lambda_{2} \widehat{v}_{0}+\frac{\lambda_{2}^{2}+a_{1}}{\mu} \widehat{\theta}_{0}\right], \\
& c_{3}=\frac{\mu}{\left(\lambda_{3}-\lambda_{2}\right)\left(\lambda_{3}-\lambda_{1}\right)}\left[a_{1} \widehat{e}_{0}-\lambda_{3} \widehat{v}_{0}+\frac{\lambda_{3}^{2}+a_{1}}{\mu} \widehat{\theta}_{0}\right] .
\end{aligned}
$$

Puting $f=0$ in formula (7), from the definition of the function $e$ we get

$$
\begin{gathered}
H:=\xi \times \widehat{u}=\cos \sqrt{a_{2}} t\left(\xi \times \widehat{u^{0}}\right)+\frac{\sin \sqrt{a_{2}} t}{\sqrt{a_{2}}}\left(\xi \times \widehat{u^{1}}\right), \\
H_{4}:=\xi \cdot \widehat{u}=-i \widehat{e} .
\end{gathered}
$$

Then $\widehat{u}$ can be expressed as follows:

$$
\widehat{u}=\frac{H \times \xi+H_{4} \cdot \xi}{|\xi|^{2}} .
$$

Hence for $f=0, g=0$ we obtain

$$
\begin{aligned}
\widehat{u}= & \left(\cos \sqrt{a_{2}} t I-\frac{\xi \otimes \xi}{|\xi|^{2}} \cos \sqrt{a_{2}} t+h_{1} \frac{\xi \otimes \xi}{|\xi|^{2}}\right) \widehat{u^{0}} \\
& +\left(\frac{\sin \sqrt{a_{2}} t}{\sqrt{a_{2}}} I-\frac{\sin \sqrt{a_{2}} t}{\sqrt{a_{2}}} \frac{\xi \otimes \xi}{|\xi|^{2}}+h_{2} \frac{\xi \otimes \xi}{|\xi|^{2}}\right) \widehat{u^{1}}+\frac{h_{3}}{|\xi|^{2}} \xi \widehat{\theta}_{0}, \\
\widehat{\theta}= & \widetilde{h}_{1} \widehat{\xi u^{0}}+\widetilde{h}_{2} \widehat{\xi u^{1}}+\widetilde{h}_{3} \widehat{\theta}_{0},
\end{aligned}
$$

where

$$
\begin{aligned}
h_{1}= & \frac{\lambda_{2} \lambda_{3}-a_{1}}{\left(\lambda_{2}-\lambda_{1}\right)\left(\lambda_{3}-\lambda_{1}\right)} e^{\lambda_{1} t}-\frac{\lambda_{2} \lambda_{3}-a_{1}}{\left(\lambda_{2}-\lambda_{1}\right)\left(\lambda_{3}-\lambda_{2}\right)} e^{\lambda_{2} t}+\frac{\lambda_{1} \lambda_{2}-a_{1}}{\left(\lambda_{3}-\lambda_{2}\right)\left(\lambda_{3}-\lambda_{1}\right)} e^{\lambda_{3} t}, \\
h_{2}= & \frac{D+\lambda_{1}}{\left(\lambda_{2}-\lambda_{1}\right)\left(\lambda_{3}-\lambda_{1}\right)} e^{\lambda_{1} t}+\frac{D+\lambda_{2}}{\left(\lambda_{2}-\lambda_{1}\right)\left(\lambda_{3}-\lambda_{2}\right)} e^{\lambda_{2} t}+\frac{D+\lambda_{3}}{\left(\lambda_{3}-\lambda_{2}\right)\left(\lambda_{3}-\lambda_{1}\right)} e^{\lambda_{3} t}, \\
h_{3}= & -i\left[\frac{\left(\lambda_{1}^{2}+a_{1}\right)\left(\lambda_{2} \lambda_{3}-a_{1}\right)}{a_{1}\left(\lambda_{2}-\lambda_{1}\right)\left(\lambda_{3}-\lambda_{1}\right)} e^{\lambda_{1} t}-\frac{\left(\lambda_{2}^{2}+a_{1}\right)\left(\lambda_{1} \lambda_{3}-a_{1}\right)}{a_{1}\left(\lambda_{2}-\lambda_{1}\right)\left(\lambda_{3}-\lambda_{2}\right)} e^{\lambda_{2} t}\right. \\
& \left.+\frac{\left(\lambda_{3}^{2}+a_{1}\right)\left(\lambda_{1} \lambda_{2}-a_{1}\right)}{a_{1}\left(\lambda_{3}-\lambda_{2}\right)\left(\lambda_{3}-\lambda_{1}\right)} e^{\lambda_{3} t}\right]
\end{aligned}
$$


and

$$
\begin{aligned}
\widetilde{h}_{1}= & i\left[\frac{\mu a_{1}}{\left(\lambda_{2}-\lambda_{1}\right)\left(\lambda_{3}-\lambda_{1}\right)} e^{\lambda_{1} t}-\frac{\mu a_{1}}{\left(\lambda_{2}-\lambda_{1}\right)\left(\lambda_{3}-\lambda_{2}\right)} e^{\lambda_{2} t}\right. \\
& \left.+\frac{\mu a_{1}}{\left(\lambda_{3}-\lambda_{2}\right)\left(\lambda_{3}-\lambda_{1}\right)} e^{\lambda_{3} t}\right], \\
\widetilde{h}_{2}= & i\left[-\frac{\mu \lambda_{1}}{\left(\lambda_{2}-\lambda_{1}\right)\left(\lambda_{3}-\lambda_{1}\right)} e^{\lambda_{1} t}+\frac{\mu \lambda_{2}}{\left(\lambda_{2}-\lambda_{1}\right)\left(\lambda_{3}-\lambda_{2}\right)} e^{\lambda_{2} t}\right. \\
& \left.-\frac{\mu \lambda_{3}}{\left(\lambda_{3}-\lambda_{2}\right)\left(\lambda_{3}-\lambda_{1}\right)} e^{\lambda_{3} t}\right], \\
\widetilde{h}_{3}= & \frac{\lambda_{1}^{2}+a_{1}}{\left(\lambda_{2}-\lambda_{1}\right)\left(\lambda_{3}-\lambda_{1}\right)} e^{\lambda_{1} t}-\frac{\lambda_{2}^{2}+a_{1}}{\left(\lambda_{2}-\lambda_{1}\right)\left(\lambda_{3}-\lambda_{2}\right)} e^{\lambda_{2} t} \\
& +\frac{\lambda_{3}^{2}+a_{1}}{\left(\lambda_{3}-\lambda_{2}\right)\left(\lambda_{3}-\lambda_{1}\right)} e^{\lambda_{3} t} .
\end{aligned}
$$

Now we construct a solution of the initial value problem (1) with null Cauchy data. We know that (cf. (7))

$$
\widehat{w}=\int_{0}^{t} \frac{\sin \left[\sqrt{a_{2}}(t-s)\right] \widehat{\operatorname{rot} f}}{\sqrt{a_{2}}} d s .
$$

To find the fundamental solution of (8) one has to solve the problem

$$
\begin{aligned}
\mathbb{Y}^{\prime} & =A \mathbb{Y}, \\
\mathbb{Y}(0) & =I,
\end{aligned}
$$

where $\mathbb{Y}=\left\{H_{i j}\right\}$ is a matrix with columns $\mathbb{Y}_{1}, \mathbb{Y}_{2}, \mathbb{Y}_{3}$. After a simple calculation we get

$$
\begin{aligned}
\mathbb{Y}_{1}= & \frac{\mu a_{1}}{\left(\lambda_{2}-\lambda_{1}\right)\left(\lambda_{3}-\lambda_{1}\right)} Y_{1}-\frac{\mu a_{1}}{\left(\lambda_{2}-\lambda_{1}\right)\left(\lambda_{3}-\lambda_{2}\right)} Y_{2}+\frac{\mu a_{1}}{\left(\lambda_{3}-\lambda_{2}\right)\left(\lambda_{3}-\lambda_{1}\right)} Y_{3}, \\
\mathbb{Y}_{2}= & -\frac{\mu \lambda_{1}}{\left(\lambda_{2}-\lambda_{1}\right)\left(\lambda_{3}-\lambda_{1}\right)} Y_{1}+\frac{\mu \lambda_{2}}{\left(\lambda_{2}-\lambda_{1}\right)\left(\lambda_{3}-\lambda_{2}\right)} Y_{2}-\frac{\mu \lambda_{3}}{\left(\lambda_{3}-\lambda_{2}\right)\left(\lambda_{3}-\lambda_{1}\right)} Y_{3}, \\
\mathbb{Y}_{3}= & \frac{\lambda_{1}^{2}+a_{1}}{\left(\lambda_{2}-\lambda_{1}\right)\left(\lambda_{3}-\lambda_{1}\right)} Y_{1}-\frac{\lambda_{2}^{2}+a_{1}}{\left(\lambda_{2}-\lambda_{1}\right)\left(\lambda_{3}-\lambda_{2}\right)} Y_{2} \\
& +\frac{\lambda_{3}^{2}+a_{1}}{\left(\lambda_{3}-\lambda_{2}\right)\left(\lambda_{3}-\lambda_{1}\right)} Y_{3} .
\end{aligned}
$$

Therefore the fundamental solution is equal to $\mathbb{H}=h(t)\left\{H_{i j}\right\}$, where $h$ denotes the Heaviside function. The solution of (8) with $G=[0, \widehat{\operatorname{div} f}, \widehat{g}]^{T}$ is

$$
Y=\int_{0}^{t}\left\{H_{i j}(t-s)\right\} G(s) d s .
$$


Let $-i \widehat{w}=S=\left[S_{1}, S_{2}, S_{3}\right]^{T}, S_{4}=-i \widehat{e}$. We have

and consequently

$$
\widehat{u}=\frac{\xi S_{4}}{|\xi|^{2}}+\frac{S \times \xi}{|\xi|^{2}}
$$

$$
\begin{aligned}
\widehat{u}= & \int_{0}^{t}\left\{\frac{\sin \left[\sqrt{a_{2}}(t-s)\right]}{\sqrt{a_{2}}} I-\frac{\xi \otimes \xi}{|\xi|^{2}} \frac{\sin \left[\sqrt{a_{2}}(t-s)\right]}{\sqrt{a_{2}}}+\frac{\xi \otimes \xi}{|\xi|^{2}} H_{12}(t-s)\right\} \widehat{f} d s \\
& +\int_{0}^{t} \frac{H_{13}(t-s)}{|\xi|^{2}}(-i) \xi \widehat{g} d s, \\
\widehat{\theta}= & \int_{0}^{t}\left[H_{32}(t-s) i \xi \widehat{f}+H_{33}(t-s) \widehat{g}\right] d s .
\end{aligned}
$$

The solution of the problem (1) is of course the sum of the solutions of (10) and (11).

For further investigation of this solution we derive the asymptotic behaviour of the roots $\lambda_{1}, \lambda_{2}$ and $\lambda_{3}$. For $|\xi| \rightarrow 0$ we get

and for $|\xi| \rightarrow \infty$,

$$
\begin{aligned}
\lambda_{1} & =\frac{-c_{1}^{2} \delta}{c_{1}^{2}+a}|\xi|^{2}+O\left(|\xi|^{4}\right), \\
\lambda_{2,3} & = \pm i \sqrt{c_{1}^{2}+a}|\xi|-\frac{a \delta}{2\left(c_{1}^{2}+a\right)}|\xi|^{2}+O\left(|\xi|^{3}\right),
\end{aligned}
$$

$$
\begin{gathered}
\lambda_{1}=-\delta|\xi|^{2}+\frac{a \delta}{c_{1}^{2} l_{1}^{2}+\delta^{2}}+O\left(|\xi|^{-1}\right), \\
\lambda_{2,3}=-\frac{a \delta}{2\left(c_{1}^{2} l_{1}^{2}+\delta^{2}\right)} \pm i c_{1}^{2} l_{1}^{2}|\xi|^{2}+O(1),
\end{gathered}
$$

where $\delta=k / c, a=T_{0} m^{2} / \varrho c$.

Proof of Theorem 1. Let $\langle\xi\rangle=\sqrt{1+|\xi|^{2}},\|\cdot\|_{L_{2}}=\|\cdot\|$ and let $C_{1}$ denote a large constant. Note that $h_{1 \mid t=0}=1$, and in view of the asymptotic behaviour and the properties of $\lambda_{1}, \lambda_{2}, \lambda_{3}$, the function $h_{1}$ is bounded. Hence

$$
\begin{aligned}
\int_{\mathbb{R}^{3}}\left|\left(\cos \sqrt{a_{2}} t I-\frac{\xi \otimes \xi}{|\xi|^{2}} \cos \sqrt{a_{2}} t+h_{1} \frac{\xi \otimes \xi}{|\xi|^{2}}\right) \widehat{u^{0}}\right|^{2}\langle\xi\rangle^{2 s} d \xi & \\
& \leq C_{1} \int\langle\xi\rangle^{2}\left|\widehat{u^{0}}\right|^{2} d \xi=C_{1}\left\|u^{0}\right\|_{s}^{2} .
\end{aligned}
$$

Note that $h_{2 \mid t=0}=0, h_{2}=h_{2}^{\prime}(\theta t) t, \theta \in(0,1)$, and hence

$$
\begin{aligned}
\int_{\mathbb{R}^{3}}\left|\frac{\sin \sqrt{a_{2}} t}{\sqrt{a_{2}}} I-\frac{\sin \sqrt{a_{2}} t}{\sqrt{a_{2}}} \frac{\xi \otimes \xi}{|\xi|^{2}}+h_{2} \frac{\xi \otimes \xi}{|\xi|^{2}}\right|^{2}\left|\widehat{u^{1}}\right|^{2}\langle\xi\rangle^{2 s} d \xi \\
\leq C_{1}\left(1+t^{2}\right) \int_{\mathbb{R}^{3}}\langle\xi\rangle^{2 s}\left|\widehat{u^{1}}\right| d \xi=C_{1}\left(1+t^{2}\right)\left\|u^{1}\right\|_{s}^{2} .
\end{aligned}
$$


Now $h_{3 \mid t=0}=0$, so

$$
\begin{aligned}
\int_{\mathbb{R}^{3}}\left|\frac{h_{3}}{|\xi|^{2}} \xi \widehat{\theta}_{0}\right|^{2}\langle\xi\rangle^{2 s} d \xi & \leq C_{1}\left(1+t^{2}\right) \int_{\mathbb{R}^{3}}\left|\xi \widehat{\theta}_{0}\right|^{2}\langle\xi\rangle^{2 s} d \xi \\
& \leq C_{1}\left(1+t^{2}\right) \int_{\mathbb{R}^{3}}\left|\widehat{\theta}_{0}\right|^{2}\langle\xi\rangle^{2 s+2} d \xi=C_{1}\left(1+t^{2}\right)\left\|\theta_{0}\right\|_{s+1}^{2} .
\end{aligned}
$$

Moreover, $\widetilde{h}_{1 \mid t=0}=0$, and therefore

$$
\int_{\mathbb{R}^{3}}\left|\widetilde{h}_{1} \widehat{\xi u^{0}}\right|^{2}\langle\xi\rangle^{2 s} d \xi \leq C_{1}\left(1+t^{2}\right)\left\|u^{0}\right\|_{s+1}^{2} .
$$

Also, $\widetilde{h}_{2 \mid t=0}=0, \widetilde{h}_{2} \lambda_{k}$ is bounded, and so

$$
\int_{\mathbb{R}^{3}}\left|\widetilde{h}_{2} \widehat{\xi u^{1}}\right|^{2}\langle\xi\rangle^{2 s} d \xi \leq C_{1}\left(1+t^{2}\right)\left\|u^{1}\right\|_{s+1}^{2} .
$$

Next, $\widetilde{h}_{3 \mid t=0}=1$, and therefore

$$
\int_{\mathbb{R}^{3}}\left|\widetilde{h}_{3} \widehat{\theta}_{0}\right|^{2}\langle\xi\rangle^{2 s} d \xi \leq\left\|\theta_{0}\right\|_{s}^{2}
$$

Since $H_{12 \mid t=0}=0$ we have

$$
\begin{aligned}
\int_{\mathbb{R}^{3}} \mid \int_{0}^{t}\left\{\sin \left[\sqrt{a_{2}}(t-s)\right] I-\frac{\xi \otimes \xi}{|\xi|^{2}} \sin \left[\sqrt{a_{2}}(t-s)\right]\right. \\
\left.\quad+\frac{\xi \otimes \xi}{|\xi|^{2}} H_{12}(t-s)\right\}\left.\widehat{f(s)} d s\right|^{2}\langle\xi\rangle^{2 s} d \xi \leq C_{1}\left(1+t^{4}\right) \sup _{\tau \in[0, t]}\|f(\tau)\|_{s}^{2},
\end{aligned}
$$

and similarly $H_{13 \mid t=0}=0$ implies

$$
\int_{\mathbb{R}^{3}}\left|\int_{0}^{t} \frac{H_{13}(t-s)}{|\xi|^{2}}(-i) \widehat{\xi g(s)} d s\right|^{2}\langle\xi\rangle^{2 s} d \xi \leq C_{1}\left(1+t^{4}\right) \sup _{\tau \in[0, t]}\|g(\tau)\|_{s+1}^{2},
$$

and $H_{32 \mid t=0}=0$ implies

$$
\int_{\mathbb{R}^{3}}\left|\int_{0}^{t} H_{32}(t-s) i \xi \widehat{f(s)} d s\right|^{2}\langle\xi\rangle^{2 s} d \xi \leq C_{1}\left(1+t^{4}\right)\|f\|_{s+1}^{2} .
$$

Finally, $H_{33}$ is bounded, and hence

$$
\int_{\mathbb{R}^{3}}\left|\int_{0}^{t} H_{33}(t-s) \widehat{g(s)} d s\right|^{2}\langle\xi\rangle^{2 s} d \xi \leq C_{1}\left(1+t^{2}\right) \sup _{\tau \in[0, t]}\|g(\tau)\|_{s+1}^{2} .
$$

The other elements of $\|u\|_{s}^{2},\|\theta\|_{s}^{2}$ can be estimated by using the inequality $a b \leq \frac{1}{2}\left(a^{2}+b^{2}\right)$. 


\section{References}

[1] G. Ahmadi and K. Firoozbakhsh, First strain-gradient theory of thermoelasticity, Int. J. Solids Structures 11 (1975), 339-345.

[2] R. D. Mindlin and N. N. Eshel, On first strain-gradient theories in linear elasticity, ibid. 4 (1968), 109-124.

[3] L. S. Pontryagin, Ordinary Differential Equations, Addison-Wesley, 1962.

[4] R. A. Toupin, Elastic materials with couple-stresses, Arch. Ration. Mech. Anal. 11 (1962), 385-414.

Institute of Mathematics and Cryptology

Faculty of Cybernetics

Military University of Technology

S. Kaliskiego 2

01-489 Warszawa, Poland

E-mail: jkolakowski@wat.edu.pl

jlazuka@wat.edu.pl

Received on 27.7.2007;

revised version on 16.3.2008 
